\title{
BMJ Open i-Move, a personalised exercise intervention for patients with advanced melanoma receiving immunotherapy: a randomised feasibility trial protocol
}

Amelia Hyatt (D) , ${ }^{1}$ Karla Gough, ${ }^{1,2}$ Andrew Murnane (D) , ${ }^{3,4}$ George Au-Yeung, ${ }^{5}$ Tamara Dawson, ${ }^{1}$ Elizabeth Pearson, ${ }^{1}$ Haryana Dhillon (D) , ${ }^{6,7}$ Shahneen Sandhu, ${ }^{5,8}$ Narelle Williams, ${ }^{9}$ Elizabeth Paton, ${ }^{9}$ Alex Billett, ${ }^{1}$ Anya Traill, ${ }^{10}$ Hayley Andersen, ${ }^{11}$ Victoria Beedle, ${ }^{12}$ Donna Milne ${ }^{1}$

To cite: Hyatt A, Gough $\mathrm{K}$, Murnane A, et al. i-Move, a personalised exercise intervention for patients with advanced melanoma receiving immunotherapy: a randomised feasibility trial protocol. BMJ Open 2020;10:e036059. doi:10.1136/ bmjopen-2019-036059

- Prepublication history for this paper is available online. To view these files, please visit the journal online (http://dx.doi. org/10.1136/bmjopen-2019036059).

Received 28 November 2019 Revised 26 January 2020 Accepted 30 January 2020

Check for updates

(C) Author(s) (or their employer(s)) 2020. Re-use permitted under CC BY-NC. No commercial re-use. See rights and permissions. Published by BMJ.

For numbered affiliations see end of article.

Correspondence to

Dr Donna Milne;

donna.milne@petermac.org

\section{ABSTRACT}

Introduction There is increasing evidence demonstrating the benefits of exercise in counteracting cancer treatmentrelated fatigue. Immunotherapy is an established treatment for advanced melanoma, and is associated with fatigue in a third of patients. The safety and efficacy of exercise in counteracting treatment-related fatigue in patients with advanced melanoma receiving immunotherapy are yet to be determined. This study aims to assess the safety, adherence to and acceptability of a mixed-methods parallel-group, pilot randomised controlled trial of a personalised, 12-week semi-supervised exercise programme prescribed by an exercise physiologist (iMove) in 30 patients with stage IV melanoma scheduled to commence immunotherapy: single agent ipilimumab, nivolumab or pembrolizumab, or combination ipilimumab and nivolumab. The trial will be used to provide preliminary evidence of the potential efficacy of exercise for managing fatigue.

Methods and analysis Thirty participants will be recruited from a specialist cancer centre between May and September, 2019. Participants will be randomised 1:1 to receive iMove, or usual care (an information booklet about exercise for people with cancer). Feasibility data comprise: eligibility; recruitment and retention rates; adherence to and acceptability of exercise consultations, personalised exercise programme and study measures; and exercise-related adverse events. Patient-reported outcome measures assess potential impact of the exercise intervention on: fatigue, role functioning, symptoms and quality of life. Follow-up will comprise five time points over 24 weeks. Physical assessments measure physical fitness and functioning.

Ethics and dissemination This study was reviewed and approved by the Peter MacCallum Cancer Centre Human Research Ethics Committee (HREC/48927/PMCC2019). The findings from this trial will be disseminated via conference presentations and publications in peerreviewed journals, and by engagement with clinicians, media, government and consumers. In particular, we will promote the outcomes of this work among the oncology community should this pilot indicate benefit for patients.

\section{Strength and limitations of this study}

- This trial will provide novel data regarding the feasibility of delivering exercise interventions to counteract immunotherapy-related fatigue in the advanced melanoma population.

- Given the success of immunotherapy as a treatment for melanoma, and many other cancers, developing an exercise intervention which counteracts immunotherapy-related fatigue has the potential to benefit many patients.

- Exercise physiologists undertaking physical assessments cannot be blinded to participation randomisation outcomes due to pragmatic design limitations.

- This is a single site study, so may not produce results that are directly relevant to all patients and all settings; well-designed multi-site trials are needed for this purpose.

Trial registration number ACTRN12619000952145; Pre-results.

\section{INTRODUCTION}

WHO estimated approximately 62000 people died from melanoma in 2015 globally ${ }^{1}$. In Australia, there were 1,190 deaths from melanoma in 2019. ${ }^{2}$ Until recently, survival rates for those diagnosed with advanced melanoma were low, however the introduction of immune checkpoint inhibitors that target programmed cell death protein 1 and cytotoxic T-lymphocyte-associated protein 4 ligands, commonly referred to as 'immunotherapy', has resulted in significant long-term survival gains for a proportion of these people. ${ }^{3-6}$ Immunotherapy can cause a variety of side effects which can impact quality of life, the most common being fatigue. ${ }^{7}$ Immunotherapy-related fatigue occurs in a third of patients ${ }^{8}$ and has been reported by 
patients as having a detrimental effect on their ability to engage in many facets of their lives, particularly caring for children and working. ${ }^{9}$

Cancer-related fatigue is a well-known side effect of anti-cancer therapies. Exercise interventions have been developed to prevent or counteract fatigue with promising results. ${ }^{10} 11$ Research into the impact of strength and resistance-based exercise on cancer and treatmentrelated symptoms has been undertaken in breast, prostate and colorectal cancer populations, during or after chemotherapy or radiotherapy. ${ }^{1011}$ The Clinical Oncology Society of Australia recently issued a position statement on exercise for cancer patients, that recommends weekly participation in both aerobic and resistance training activities. ${ }^{12}$ Importantly, the statement in combination with current research supports that generic prescription of exercise therapy is safe, tolerable and efficacious for improving symptoms experienced by patients with cancer, both during and after anti-cancer therapy. ${ }^{11} 13$ However, few empirical evaluations of the role of exercise in combating symptoms of the cancer or side effects of treatment have included patients either with melanoma or those receiving immunotherapy. A feasibility study in patients with stage IV melanoma where exercise was prescribed as part of a broader supportive care intervention, indicated exercise was acceptable and achievable for patients receiving single agent immunotherapy. ${ }^{14}$

Given the rapid expansion of immunotherapy into mainstream treatment of melanoma, and increasingly other cancers, exercise interventions which effectively reduce immunotherapy-related fatigue have the potential to benefit many patients. The iMove programme, a 12-week personalised exercise physiologist (EP)-prescribed exercise programme, has therefore been developed for patients diagnosed with advanced melanoma receiving immunotherapy. The primary aims of this pilot trial are to assess the: (i) safety and acceptability of the iMove programme to patients, and (ii) feasibility of conducting a definitive randomised controlled trial (RCT). Second, the trial will provide preliminary evidence of efficacy of an exercise intervention to combat fatigue, as well as other measures of interest.

\section{METHODS}

\section{Trial design and setting}

The trial is a mixed-methods parallel-group, randomised controlled pilot trial. Qualitative and quantitative methods will be used. This study will be run at a specialist cancer hospital in Melbourne, Australia.

\section{Patient and public involvement}

The i-Move pilot trial is the product of consumer-driven research. Melanoma Patients Australia recently sought advice from our group about the potential for exercise to ameliorate immunotherapy-induced fatigue, as patients and families had been asking for advice, and for research to be conducted in the area. Our project steering committee comprises a consumer advocate to advise on project design, operations and materials, specifically to assess the burden of the intervention and participation requirements.

\section{Participants}

Patients diagnosed with stage IV melanoma scheduled to receive, or recently commenced, combination or single agent immunotherapy (ipilimumab, nivolumab, pembrolizumab) will be invited to participate. Specific eligibility criteria include: aged 18 years or older, Eastern Cooperative Oncology Group (ECOG) performance status of $0-2$, not yet received second infusion of immunotherapy, able to speak and read English sufficiently to complete questionnaires and take part in the exercise programme without use of interpreter, deemed suitable to take part in an exercise intervention by their treating clinician (i.e. no medical contraindications to exercise), and have access to a smartphone.

Exclusion criteria comprises the following contraindications to safe exercise: grade $\geq 3$ peripheral neuropathy, a recent history (within the previous 3 months) of myocardial infarction or unstable angina, cerebrovascular event or transient ischaemic attack or pulmonary embolic event, or existing acute or chronic deep vein thrombosis, or active sepsis.

All interested eligible patients will be requested to provide written informed consent.

\section{Physical and safety assessment}

Consented patients will complete a general health and medical safety assessment with an EP to determine trial eligibility (as detailed earlier). Data generated from the safety assessment will also be used to identify any comorbidities or other health considerations which will need to be factored into exercise prescription for the intervention group.

Once eligibility is confirmed, the EP will complete a baseline physical assessment using standard clinical exercise tests measuring cardiovascular fitness, upper limb strength and lower limb strength (see table 1 for further information on assessments). Outcomes will be used by the EP to develop a personalised exercise programme for participants randomised to the intervention group. The EP will also determine whether the participant meets the Clinical Oncology Society of Australia guidelines for activity in people with cancer ${ }^{12}$ :

- At least $150 \mathrm{~min}$ of moderate intensity or $75 \mathrm{~min}$ of vigorous-intensity aerobic exercise (eg, walking, jogging, cycling, swimming) each week; and

- Two to three resistance exercise (ie, lifting weights) sessions each week involving moderate to vigorousintensity exercises targeting the major muscle groups.

Participants will be stratified at randomisation according to whether they meet these guidelines or not. 
Table 1 Participant data collection will occur at five time points throughout the trial

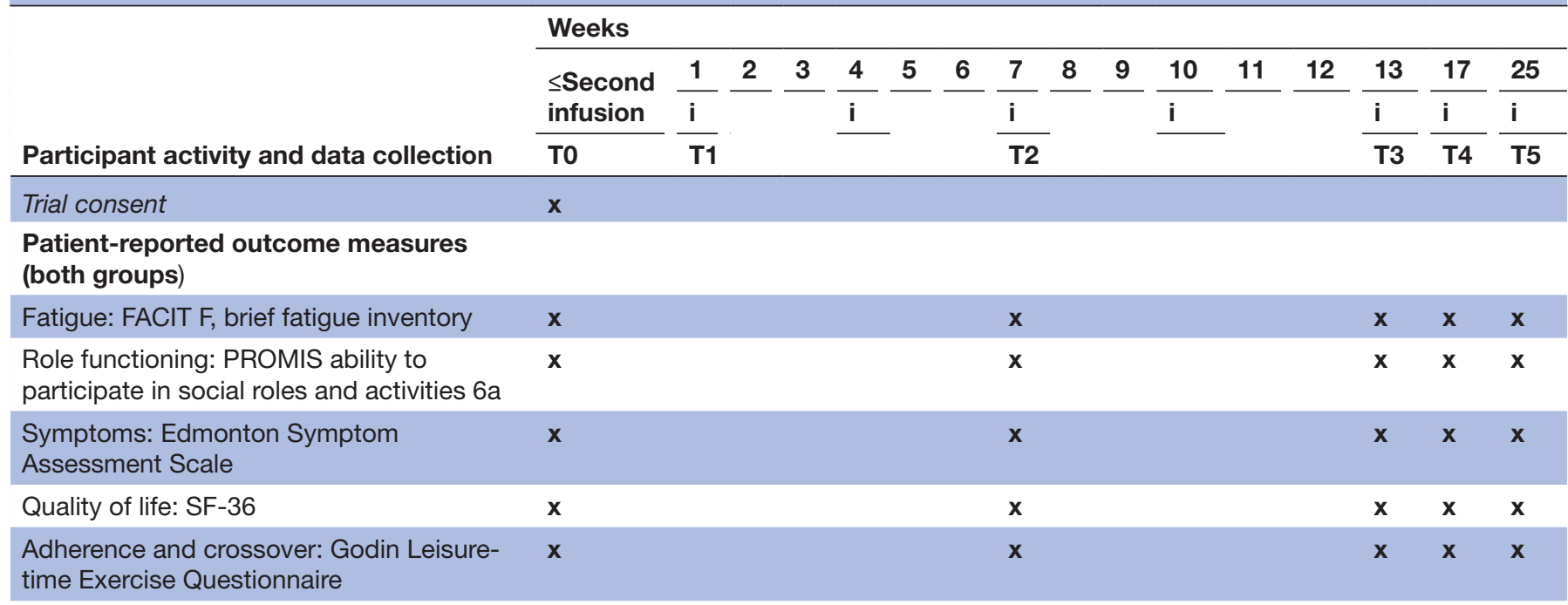

\section{Safety assessment (both groups)}

Peripheral neuropathy and other health comorbidities

\section{$\mathbf{x}$}

\section{Physical assessments (both groups)}

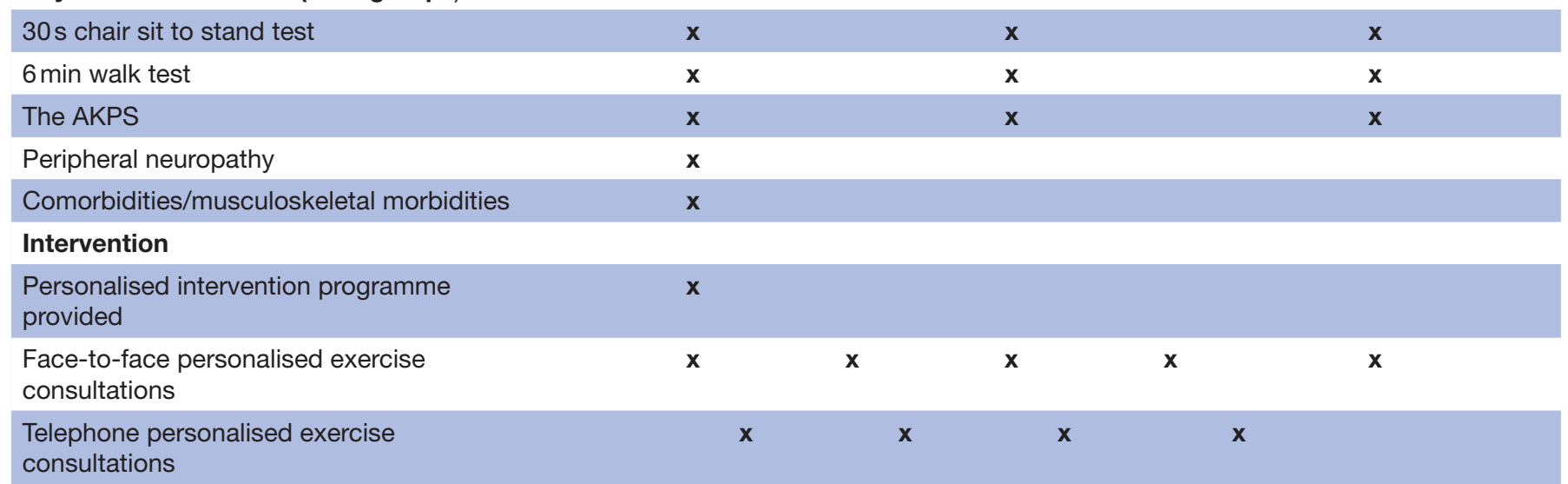

\section{Active control}

Cancer council exercise for people living with cancer booklet provided

EP consultation

\section{Feasibility measures}

Project operations (accrual, adherence, $\quad$ x retention)

\begin{tabular}{|c|c|c|c|c|c|c|c|c|c|c|}
\hline $\begin{array}{l}\text { Qualitative intervention patient interview } \\
\text { (adherence, acceptability) }\end{array}$ & & & & & & & & & $\mathbf{x}$ & \\
\hline Exercise-related adverse event log (safety) & $\mathbf{x}$ & $\mathbf{x}$ & $\mathbf{x}$ & $\mathbf{x}$ & $\mathbf{x}$ & $\mathbf{x}$ & $\mathbf{x}$ & $\mathbf{x}$ & $\mathbf{x}$ & \\
\hline
\end{tabular}
adherence, fidelity)

${ }^{*} x$ indicates event is scheduled to occur AKPS, Australia-modified Karnofsky Performance Scale; EP, exercise physiologist; FACIT-F, Functional assessment of chronic illness therapy-fatigue; SF-36, Short Form 36 Health Survey Questionnaire.

Randomisation and allocation concealment

Participants will be randomised 1:1 to either the intervention or control group. The research team will perform randomisation and notification of randomisation outcome to both patients and the EP. Randomisation will be performed using a computer-generated block allocation sequence (block size of 6 ), stratified by current exercise activity: whether meeting Clinical Oncology Society 


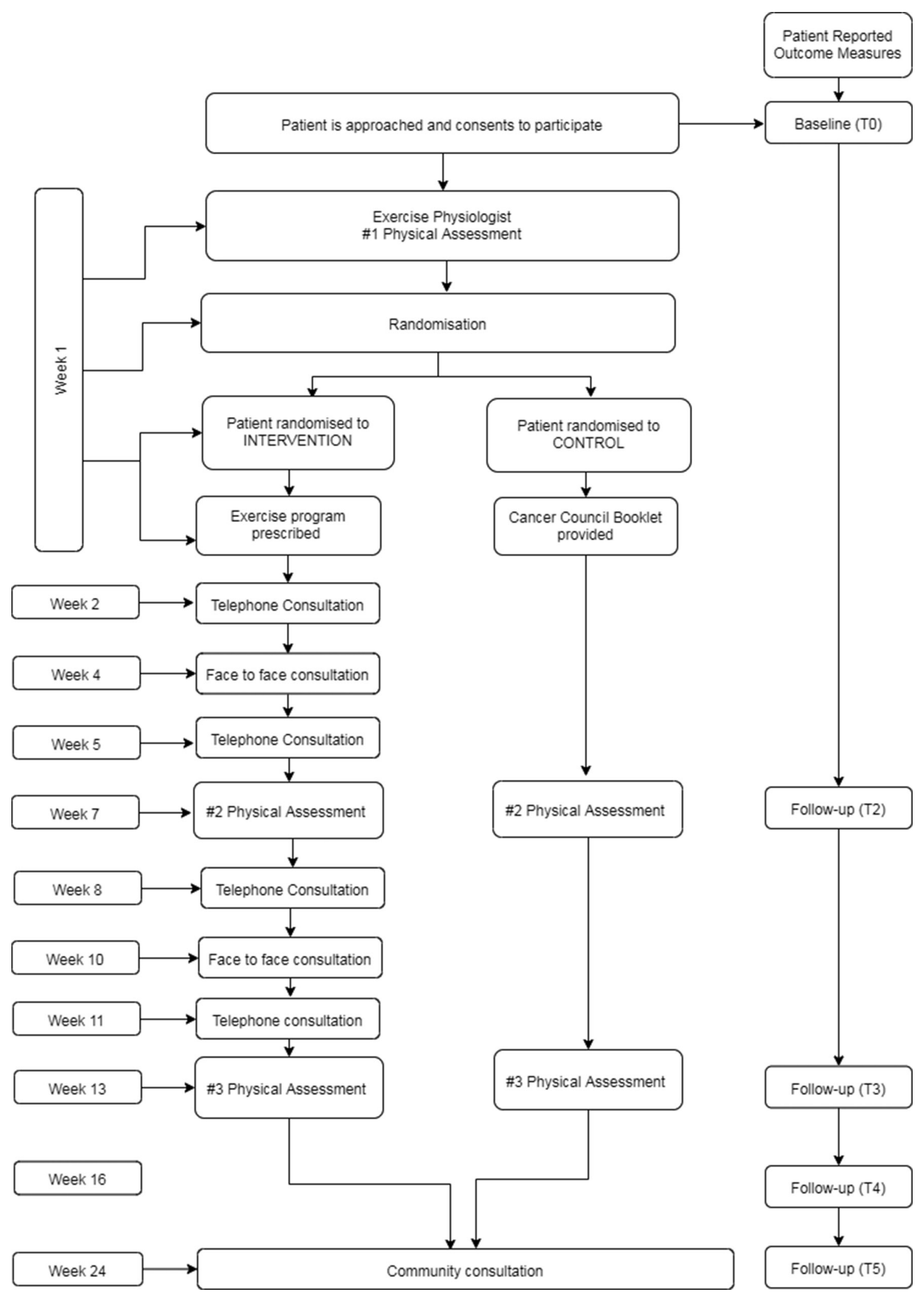

Figure 1 iMove study flow chart.

of Australia guidelines for activity in people with cancer. Allocation will be concealed from participants until after they have completed baseline physical and safety assessment and baseline patient-reported outcome measures . For those randomised to the intervention group, the EP will commence development and prescription of their exercise programme (see figure 1).

\section{Sample size}

The target sample is 30 participants randomised, 15 per group. The target sample is pragmatic, based on available funds and trial time frames; however, stepped rules of thumb for pilot sample sizes were considered. ${ }^{1516}$ If 30 patients are accrued in 3 months, then the expected monthly accrual rate is 10 patients per month with an exact $95 \% \mathrm{CI}$ of 6.75 to 
14.3 patients per month, corresponding to a $95 \%$ CI for the accrual rate over 3 months of 20.2 to 42.8 patients. Power will be insufficient to detect minimum clinically important differences between trial arms on patient-reported outcomes measures; however, if the 95\% CIs for trial arm comparisons at follow-ups T3 through T5 (see table 1) contain important differences, the intervention will be viewed as promising. ${ }^{15}$

\section{Intervention}

The intervention will be delivered by an accredited EP based in the health service. Each individual programme will be designed by the EP, accounting for relevant demographic and clinical characteristics (eg, disease, age), physical suitability, any safety considerations and current exercise activities including work, home and leisure. The EP will use the Physitrack smartphone application (app) to provide each participant with their exercise programme. Physitrack is free for patients to download to their smartphone. Participants are instructed to use the app to view and log exercise activities completed, but not change the prescribed exercise programme. EP guidance provided as part of the exercise intervention will comprise:

- Four face-to-face sessions at the hospital: once every 3 weeks (or every 2-4 weeks for those receiving nivolumab) coinciding with immunotherapy infusion appointments.

- Four telephone supervision sessions scheduled for the week after the face-to-face session.

- One final face-to-face session at the conclusion of the exercise intervention to discuss ongoing physical activity and community resources patients can access close to home.

Each participant will be prescribed an individualised exercise programme which includes moderate intensity aerobic exercise, resistance training exercises and stretching of the major muscle groups. The most common forms of exercise which will be prescribed will be walking and cycling. As per the American College of Sports Medicine and Exercise and Sports Science Australia guidelines the aim is for aerobic exercise to be undertaken 3-5 times per week (20-45 min duration) with resistance training performed 2-3 times per week. ${ }^{1718}$ Clinical reasoning will be used during the follow-up appointments to adjust the exercise intensity depending on treatment symptoms and side effects experienced by the participant.

Aerobic training heart rate range goal will be between $50 \%$ and $80 \%$ of predicated heart rate max and rating of perceived exertion on Borg's scale, with participants asked to maintain intensity between 12 and 15 (out of 20) throughout the session. Strength training will consist of 5-7 upper and lower limb exercises of the major muscle groups, starting at two sets of 10 repetitions. Strength training exercises incorporate body weight exercises, dumbbells and barbells. Initial progression of the programme involves increasing the number of repetitions per set from 10 to 12 to 15 , then increasing sets from 2 to 3 . Following increase in sets, weight is added for further resistance
During both telephone and face-to-face sessions, the EP will monitor safety and exercise progress, and update the exercise prescription if required.

\section{Control}

Participants randomised to receive the active control will be provided with a copy of the Cancer Council New South Wales booklet 'Exercise for people living with cancer' which provides general information on exercise for people with cancer. ${ }^{19}$ After 24 weeks, participants in the control group will receive a face-to-face session with the EP to provide information about accessing communitybased exercise supports via their general practitioner.

\section{Outcomes}

Study outcomes were designed to determine whether a multi-site efficacy RCT is feasible. Table 2 details each outcome, objective, data collection method and where relevant, pre-specified feasibility criteria.

\section{Data collection and management}

Data pertaining to each study outcome will be collected and recorded usingfour main methods: project operations database, custom EP data collection instrument, patientreported outcome measures, and qualitative interviews. Data relating to iMove impact (patient-reported outcome measures and physical assessment) will be collected across five time points, outlined in detail in table 1.

The project operations database is designed to collect data as per Consolidated Standards of Reporting Trials (CONSORT) guidelines ${ }^{20}$ and feasibility outcomes, namely recruitment and ineligibility. The EP-log is a custom-built workbook designed for the EPs to record safety and physical assessment outcomes, and to document prescribed exercise programme activities, consultation (face-to-face and telephone) discussions, exercise-related adverse events and adherence and acceptability data. Additional feasibility outcome data regarding acceptability and adherence will be collected via participant and EP qualitative interviews at the conclusion of trial participation.

Physical assessment data will be collected and recorded in the EP data collection instrument using the 30 s chair stand test ${ }^{21}$; the 6 min walk test ${ }^{22}$; the arm curl test ${ }^{23}$ and the Australia-modified Karnofsky Performance Scale. ${ }^{24}$

Semi-structured interviews will be undertaken by the research assistant 1:1 with the EP and participant randomised to the intervention group. The interview will comprise questions on the EP experience and perspectives regarding the feasibility and acceptability of the intervention, barriers and facilitators to adherence to the exercise programme in particular immunotherapyrelated fatigue and what could be improved if the intervention was part of a larger RCT.

Patient-reported outcome measures will be emailed to participants to complete using the REDCap data management programme,${ }^{25}$ and will comprise: the functional assessment of chronic illness therapy; brief fatigue inventory, Patient-Reported Outcomes Measurement 
Table 2 Outcomes, data collection method and feasibility criteria

\begin{tabular}{llll} 
Outcome & Objective & Data collection & Feasibility criteria \\
\hline Feasibility & & & \\
Accrual & &
\end{tabular}

\begin{tabular}{|c|c|c|c|}
\hline & $\begin{array}{l}\text { Estimate the recruitment rate and } \\
\text { appraise reasons for ineligibility }\end{array}$ & Project operation database & $\begin{array}{l}\text { On average, we will recruit } 10 \\
\text { participants per month for } 3 \text { months }\end{array}$ \\
\hline \multicolumn{4}{|l|}{ Adherence } \\
\hline
\end{tabular}

\begin{tabular}{|c|c|c|c|}
\hline & $\begin{array}{l}\text { Investigate adherence to a } \\
\text { personalised, prescribed exercise } \\
\text { intervention. }\end{array}$ & $\begin{array}{l}\text { Recorded by EP; Participant } \\
\text { qualitative interview, Physitrack }\end{array}$ & $\mathrm{N} / \mathrm{a}$ \\
\hline \multicolumn{4}{|l|}{ Acceptability } \\
\hline & $\begin{array}{l}\text { Investigate the acceptability of the EP } \\
\text { consultation sessions. }\end{array}$ & $\begin{array}{l}\text { Participant and EP qualitative } \\
\text { interview }\end{array}$ & $\mathrm{N} / \mathrm{a}$ \\
\hline & $\begin{array}{l}\text { Investigate the acceptability of a } \\
\text { personalised, prescribed exercise } \\
\text { intervention }\end{array}$ & $\begin{array}{l}\text { Recorded by EP; Participant and EP } \\
\text { qualitative interview }\end{array}$ & $\mathrm{N} / \mathrm{a}$ \\
\hline & $\begin{array}{l}\text { Assess the acceptability and } \\
\text { appropriateness of study measures }\end{array}$ & Participant qualitative interview & $\mathrm{N} / \mathrm{a}$ \\
\hline \multicolumn{4}{|l|}{ Retention } \\
\hline & $\begin{array}{l}\text { Assess the retention of participants at } \\
16 \text { weeks }\end{array}$ & Recorded by EP & $\begin{array}{l}\text { At least } 70 \% \text { of participants will } \\
\text { complete their physical assessment } \\
\text { at T3 }\end{array}$ \\
\hline \multicolumn{4}{|l|}{ Safety } \\
\hline & $\begin{array}{l}\text { Determine the number and type of } \\
\text { exercise-related adverse events }\end{array}$ & Recorded by EP & $\mathrm{N} / \mathrm{a}$ \\
\hline \multicolumn{4}{|l|}{ Impact } \\
\hline & $\begin{array}{l}\text { Investigate the potential impact of } \\
\text { iMove on fatigue and other patient- } \\
\text { reported outcomes }\end{array}$ & $\begin{array}{l}\text { Patient-reported outcome measures: } \\
\text { FACIT-F } \\
\text { BFI } \\
\text { PROMIS ability to participate in } \\
\text { aocial roles and activities - SF } 6 a \\
\text { SF-36 } \\
\text { - ESAS } \\
\text { GLTPAQ }\end{array}$ & $\mathrm{N} / \mathrm{a}$ \\
\hline
\end{tabular}

BFI, Brief fatigue inventory; EP, exercise physiologist; ESAS, Edmonton Symptom Assessment Scale; FACIT-F, Functional assessment of chronic illness therapy-fatigue; GLTPAQ, Godin Leisure-time Physical Activity Questionnaire; PROMs, patient-reported outcome measures; SF-36, Short Form 36 Health Survey Questionnaire; SF, Short Form.

Information System (PROMIS) V.2.0-ability to participate in social roles and activities 6a; Short Form 36 Health Survey Questionnaire; the revised Edmonton
Symptom Assessment Scale; the Godin Leisure-time Physical Activity Questionnaire (see table 3 for more information). 


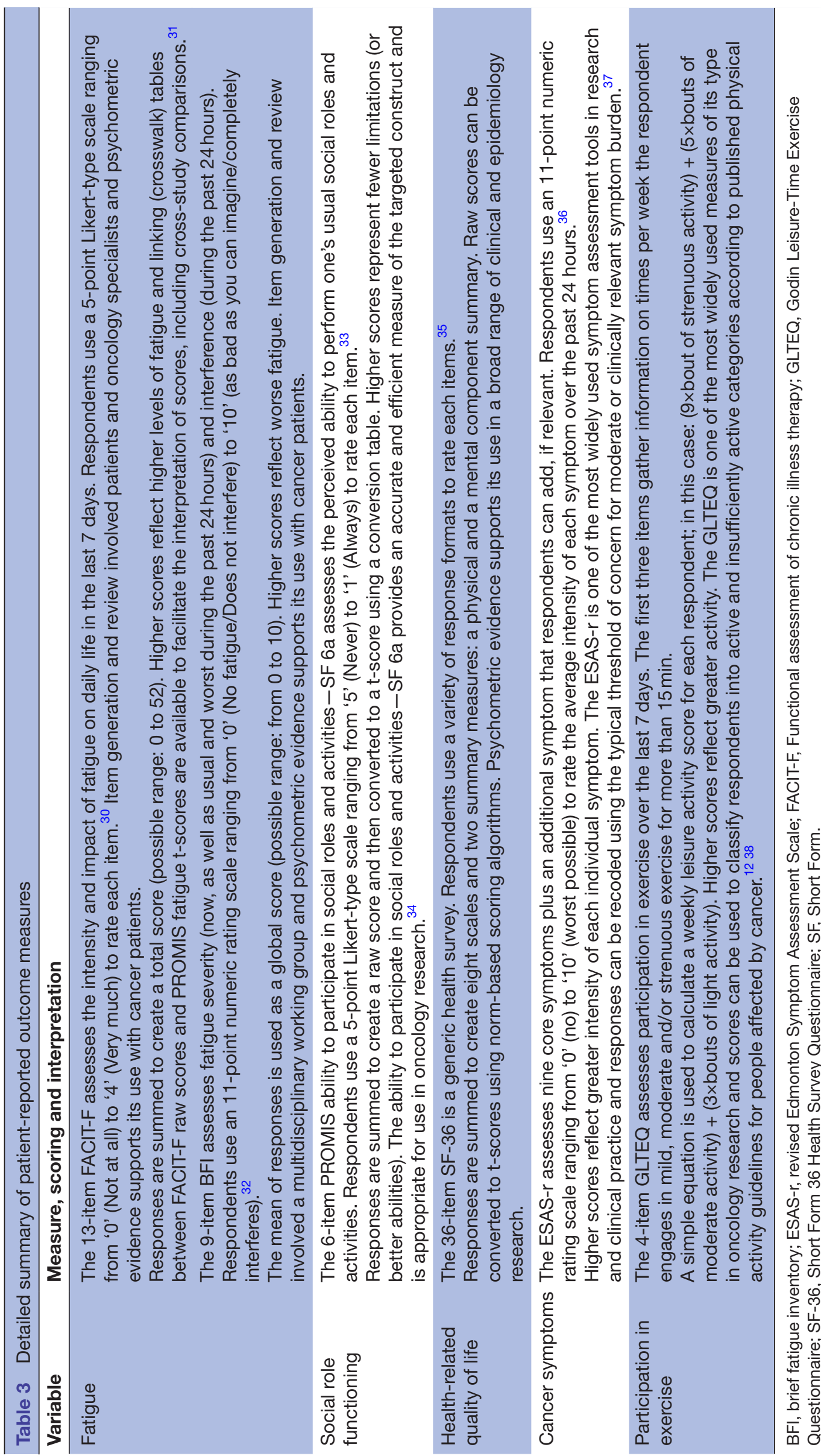




\section{Data analysis plan}

Feasibility outcomes

The main feasibility outcomes are recruitment and retention. The main acceptability outcome is adherence to the iMove consultation sessions. Recruitment data will be summarised using a rate and 95\% CI using the Poisson distribution. ${ }^{7}$ Adherence and retention data will be summarised using a proportion and 95\% CI; the latter will be estimated using the Wilson method. ${ }^{26}$ The main feasibility outcomes will be judged against prespecified criteria (table 2). Time spent by the EPs completing intervention-related activity will be recorded in the EP data collection instrument, including total time spent per patient and activity-specific time such as intervention preparation. Means and SD will be used to summarise time data. Counts and percentages will be used to summarise data on exercise-related adverse events.

Counts and percentages will be used to summarise data on missing items and forms or tests for patient-reported outcome measures and objective measures of fitness, respectively. Patient-reported outcome measures will be scored according to author guidelines. Means, SD and ranges will be used to summarise scores for patient-reported outcome measures and objective measures of fitness at each time point by trial arm. Analysis will include all available data. Values for missing forms (or measures) will not be imputed.

Analysis of patient-reported outcome measures data and objective measures of fitness will be carried out by fitting a linear mixed model to each outcome separately. Models will include fixed effects for trial arm, time and a trial arm-by-time interaction, as well as a random participant effect. Baseline assessment will be included as a covariate. Differences between trial arms at post-baseline assessments will be calculated from these models. ${ }^{27}$

\section{Qualitative data}

Data recorded by EPs (described in table 1) will be analysed using content analysis methodology. Content analysis allows for exploration of phenomenon such as motivation, experiences and views of participants in order to answer clinically relevant health questions. ${ }^{28}$ Data will be classified into codes, categories and (where relevant) themes using an iterative process. At the conclusion of analysis, findings will be presented and discussed with members of the project team. Discrepancies will be discussed and resolved until consensus with the coding framework is reached.

Interviews with EP's and patients will be audio-recorded, transcribed and analysed using interpretive description methodology. ${ }^{29}$ Interpretive description was selected for analysis as it is useful for exploring experiential data as it allows for deeper and richer thematic exploration of the data rather than sorting and summing common statements, and provides practical outcomes for clinical health services improvement. $^{29}$

\section{Data management}

Demographic, questionnaire and clinical data will be recorded on hard-copy forms, and then entered into a database built and stored on the hospital REDCap platform. Only key project personnel including the research assistant, data manager, principal investigator and project manager will have access to hard-copy and electronic data, in accordance with the National Statement on Ethical Conduct in Human Research 2007 (updated 2018) and the Australian Code for Responsible Conduct of Research 2018. During the data collection period, regular quality assurance will be undertaken to ensure accuracy, precision and completeness of trial data. At the conclusion of data collection, export and analysis, the project will be archived on the REDCap platform with backups stored as password-protected files on hospital secure servers, and hard-copy forms will be placed in secure storage. Five years after publication or dissemination of project outcomes, hard-copy and electronic data will be destroyed.

\section{Dissemination}

The findings from this trial will be disseminated via normal academic channels such as conference presentations and publications in peer-reviewed journals but also by engagement with clinicians, media, government and consumers. In particular, we will seek to promote the outcomes of this work among the broader oncology community around the benefits of exercise for patients receiving immunotherapy. Results from this work will also be fed back to consumers via our partner patient advocacy groups, and via trial participants. Appropriate publications will be identified and planned by the project steering committee but will include a publication of the protocol design, results based on the main protocol using the trial group name, and subsequent publications of data subsets only if appropriate.

\section{DISCUSSION}

This feasibility study will provide preliminary data on the safety of exercise in people with melanoma on immunotherapy. This data will provide practitioners reassurance they can supervise exercise to mediate cancer-related fatigue in this population, provided an initial assessment is first undertaken, and the exercise prescription tailored to the individual participant. It will also provide some indication of the likely effect of exercise on a range of physical and psycho-social endpoints.

\section{Author affiliations}

${ }^{1}$ Department of Cancer Experiences Research, Peter MacCallum Cancer Centre, Melbourne, Victoria, Australia

${ }^{2}$ Department of Nursing, The University of Melbourne, Melbourne, Victoria, Australia ${ }^{3}$ ONTrac at Peter Mac Victorian Adolescent and Young Adult Cancer Service, Peter MacCallum Cancer Centre, Melbourne, Victoria, Australia

${ }^{4}$ Institute for Physical Activity and Nutrition (IPAN), School of Exercise and Nutrition Sciences, Deakin University Faculty of Health, Burwood, Victoria, Australia ${ }^{5}$ Department of Medical Oncology, Peter MacCallum Cancer Centre, Melbourne, Victoria, Australia

${ }^{6}$ Centre for Medical Psychology and Evidence-based Decision-making, School of Psychology, The University of Sydney, Sydney, New South Wales, Australia ${ }^{7}$ Psycho-Oncology Cooperative Research Group (POCOG), School of Psychology, Faculty of Science, The University of Sydney, Sydney, New South Wales, Australia ${ }^{8}$ Sir Peter MacCallum Department of Oncology, University of Melbourne, Melbourne, Victoria, Australia 
${ }^{9}$ Melanoma and Skin Cancer Trials Ltd, Monash University, Melbourne, Victoria, Australia

${ }^{10}$ Occupational Therapy and Physiotherapy Department, Peter MacCallum Cancer Centre, Melbourne, Victoria, Australia

${ }^{11}$ Bristol-Myers Squibb Australia, Melbourne, Victoria, Australia

${ }^{12}$ Melanoma Patients Australia, Brisbane, Queensland, Australia

Acknowledgements We would like to acknowledge and thank our consumer representative for their guidance in preparing this protocol, and the support from Cancer Experiences Research Department at Peter MacCallum Cancer Centre.

Contributors DM, $A H, K G$ and $A M$ conceptualised the study and drafted the intervention; GA-Y, TD, HD, SS, HA, EPa, AB, NW, EPe and AT reviewed draft study and intervention design and provided critical feedback to strengthen/amend to final design. All authors were involved in planning the methods and measurement selection and apriori analysis planning. AH and KG wrote initial draft of the paper; all authors reviewed and provided comprehensive contribution to final paper.

Funding This study was funded by Peter MacCallum Cancer Foundation (\#1832). Competing interests None declared.

Patient consent for publication Not required.

Provenance and peer review Not commissioned; externally peer reviewed.

Open access This is an open access article distributed in accordance with the Creative Commons Attribution Non Commercial (CC BY-NC 4.0) license, which permits others to distribute, remix, adapt, build upon this work non-commercially, and license their derivative works on different terms, provided the original work is properly cited, appropriate credit is given, any changes made indicated, and the use is non-commercial. See: http://creativecommons.org/licenses/by-nc/4.0/.

\section{ORCID iDs}

Amelia Hyatt http://orcid.org/0000-0003-2322-7817

Andrew Murnane http://orcid.org/0000-0002-5992-9311

Haryana Dhillon http://orcid.org/0000-0003-4039-5169

\section{REFERENCES}

1 Anon. Global health estimates 2015: deaths by cause a, sex, by country and by region, 2000-2015. Geneva: World Health Organization, 2016.

2 Australian Institute of Health and Welfare. Cancer in Australia 2019. cancer series no.119. cat. No. can 123. Canberra: AlHW, 2019.

3 Schadendorf D, Hodi FS, Robert C, et al. Pooled analysis of long-term survival data from phase II and phase III trials of ipilimumab in unresectable or metastatic melanoma. J Clin Oncol 2015;33:1889-94.

4 Hodi FS, O'Day SJ, McDermott DF, et al. Improved survival with ipilimumab in patients with metastatic melanoma. $N$ Engl J Med 2010;363:711-23.

5 Ribas A, Wolchok JD. Cancer immunotherapy using checkpoint blockade. Science 2018;359:1350-5.

6 Wolchok JD, Chiarion-Sileni V, Gonzalez R, et al. Updated results from a phase III trial of nivolumab (NIVO) combined with ipilimumab (IPI) in treatment-naive patients (PTS) with advanced melanoma (MEL) (CheckMate 067). J Clin Oncol 2016;34:9505.

7 Carreau N, Kannan R, Madden KM, et al. Immunotherapy efficacy and safety in elderly cutaneous malignancy patients. J Clin Oncol 2018;36:e21524.

8 Larkin J, Chiarion-Sileni V, Gonzalez R, et al. Combined nivolumab and ipilimumab or monotherapy in untreated melanoma. $N$ Engl J Med 2015;373:23-34.

9 Milne D, Hyatt A, Billett A, et al. Exploring the experiences of people treated with immunotherapies for advanced melanoma and those caring for them. Cancer Nurs 2019;9000:1.

10 Cormie P, Zopf EM, Zhang X, et al. The impact of exercise on cancer mortality, recurrence, and treatment-related adverse effects. Epidemiol Rev 2017;39:71-92.

11 Dennett AM, Peiris CL, Shields N, et al. Moderate-intensity exercise reduces fatigue and improves mobility in cancer survivors: a systematic review and meta-regression. J Physiother 2016;62:68-82.

12 Clinical Oncology Society of Australia. COSA position statement on exercise in cancer care 2017.

13 Jones LW, Eves ND, Scott JM. Bench-to-Bedside approaches for personalized exercise therapy in cancer. paper presented at:
American Society of clinical oncology educational book. American Society of clinical oncology. meeting, 2017.

14 Lacey J, Lomax AJ, McNeil C, et al. A supportive care intervention for people with metastatic melanoma being treated with immunotherapy: a pilot study assessing feasibility, perceived benefit, and acceptability. Support Care Cancer 2019;27:1497-507.

15 Bell ML, Whitehead AL, Julious SA. Guidance for using pilot studies to inform the design of intervention trials with continuous outcomes. Clin Epidemiol 2018;10:153-7.

16 Whitehead AL, Julious SA, Cooper CL, et al. Estimating the sample size for a pilot randomised trial to minimise the overall trial sample size for the external pilot and main trial for a continuous outcome variable. Stat Methods Med Res 2016;25:1057-73.

17 Hayes SC, Spence RR, Galvão DA, et al. Australian association for exercise and sport science position stand: optimising cancer outcomes through exercise. J Sci Med Sport 2009;12:428-34.

18 Murnane A, Gough K, Thompson K, et al. Adolescents and young adult cancer survivors: exercise habits, quality of life and physical activity preferences. Support Care Cancer 2015;23:501-10.

19 Bruce J. Cancer Council NSW. Exercise for people living with cancer: a guide for people with cancer, their families and friends. (C) Cancer Council Australia, 2016.

20 Eldridge SM, Chan CL, Campbell MJ, et al. CONSORT 2010 statement: extension to randomised pilot and feasibility trials. Pilot Feasibility Stud 2016;2:64.

21 Jones CJ, Rikli RE, Beam WC. A 30-S chair-stand test as a measure of lower body strength in community-residing older adults. Res $Q$ Exerc Sport 1999;70:113-9.

22 Rikli RE, Jones CJ. The reliability and validity of a 6-minute walk test as a measure of physical endurance in older adults. J Aging Phys Act 1998;6:363-75.

23 Różańska-Kirschke A, Kocur P, Wilk M, et al. The Fullerton fitness test as an index of fitness in the elderly. Medical Rehabilitation 2006;10:9-16.

24 Abernethy AP, Shelby-James T, Fazekas BS, et al. The Australiamodified Karnofsky Performance Status (AKPS) scale: a revised scale for contemporary palliative care clinical practice [ISRCTN81117481]. BMC Palliat Care 2005;4:7.

25 Harris PA, Taylor R, Thielke R, et al. Research electronic data capture (REDCap)--a metadata-driven methodology and workflow process for providing translational research informatics support. J Biomed Inform 2009:42:377-81.

26 Brown LD, Cai TT, DasGupta A. Interval estimation for a binomial proportion. Statistical science 2001:101-17.

27 Fitzmaurice GM, Laird NM, Ware JH. Applied longitudinal analysis. Vol 998. John Wiley \& Sons, 2012.

28 Forman J, Damschroder L. Qualitative content analysis. In: Empirical methods for bioethics: a primer. Emerald Group Publishing Limited, 2007: 39-62.

29 Thorne S, Kirkham SR, O'Flynn-Magee K. The analytic challenge in interpretive description. Int J Qual Methods 2004;3:1-11.

30 Yellen SB, Cella DF, Webster K, et al. Measuring fatigue and other anemia-related symptoms with the functional assessment of cancer therapy (fact) measurement system. J Pain Symptom Manage 1997;13:63-74.

31 Lai J-S, Cella D, Yanez B, et al. Linking fatigue measures on a common reporting metric. J Pain Symptom Manage 2014;48:639-48.

32 Mendoza TR, Wang XS, Cleeland CS, et al. The rapid assessment of fatigue severity in cancer patients: use of the brief fatigue inventory. Cancer 1999;85:1186-96.

33 Northwestern University. PROMIS ability to participate in social roles and activities scoring manual, 2020. Available: http://www. healthmeasures.net/promis-scoring-manuals

34 Garcia SF, Cella D, Clauser SB, et al. Standardizing patient-reported outcomes assessment in cancer clinical trials: a patient-reported outcomes measurement information system initiative. J Clin Oncol 2007;25:5106-12.

35 Ware JE. SF-36 health survey update. Spine 2000;25:3130-9.

36 Hui D, Bruera E. The Edmonton Symptom Assessment System 25 Years years later: past, present, and future developments. J Pain Symptom Manage 2017;53:630-43.

37 Oldenmenger WH, de Raaf PJ, de Klerk C, et al. Cut points on 0-10 numeric rating scales for symptoms included in the Edmonton symptom assessment scale in cancer patients: a systematic review. J Pain Symptom Manage 2013;45:1083-93.

38 Amireault S, Godin G, Lacombe J, et al. Validation of the GodinShephard leisure-time physical activity questionnaire classification coding system using accelerometer assessment among breast cancer survivors. J Cancer Surviv 2015;9:532-40. 